regional vereinbarter Praxisbesonderheiten auch auf besondere „Prüfgegenstände" verwiesen. So sollen weiterhin insbesondere Verordnungen von Ärzten unter die Lupe genommen werden, die an Anwendungsbeobachtungen teilnehmen (auch ein Baustein des bisherigen $\$ 106$ SGB V, der ab 2017 nicht mehr im Gesetzestext auftaucht). Wichtigster Passus im Anhang ist jedoch die Vorgabe, dass die Wirtschaftlichkeit der Arzneimittelverordnung künftig anhand der regionalen Arzneimittelvereinbarung durchdekliniert werden kann. „Hierfür kann auch die Erfüllung von Zielkriterien auf Basis eines Katalogs für eine indikationsgerechte wirtschaftliche Wirkstoffauswahl in versorgungsrelevanten Indikationen herangezogen werden“, heißt es. Das erinnert an die „Wirkstoffvereinbarung“, mit der die KV
Bayerns als erste KV Richtgrößen durch fachgruppenspezifische Generika- und Leitsubstanzquoten ersetzt hat. Seit dem Quartal III/2015 sind diese Vorgaben für Vertragsärzte der KV Bayern verpflichtend. Um wirtschaftlich zu verordnen, müssen sie nur in den für sie relevanten Produktgruppen auf ihre Quote kommen. Um Mengen und Preise der verordneten Präparate müssen sie sich keine Gedanken mehr machen. Kritik an diesem Modell gibt es jedoch schon: Generikaquoten von über $90 \%$, wie sie in Bayern beispielsweise für Psychoanaleptika vorgesehen sind, lassen kaum noch Raum für patentgeschützte Neuheiten. Dadurch drohten auch positive Nutzenbescheide des G-BA ins Leere zu laufen, wenn Präparate trotz Zusatznutzen kaum oder gar nicht mehr Eingang in die Versorgung finden. Christoph Winnat

\section{Behandlungsfehler: Handyfotos als Beweis}

Mit einem Handy in der Arztpraxis aufgenommene Bilder können unter Umständen einen medizinischen Behandlungsfehler beweisen.

Das Oberlandesgericht Oldenburg erkannte vor Kurzem Handy-Fotos als Nachweis für den verzögerten Behandlungsbeginn einer Hirnhautentzündung an (Aktenzeichen: 5 U 156/13). Im konkreten Fall ließ das Oberlandesgericht das Handy durch einen Sachverständigen auswerten, der bestätigte, dass die sich darauf befindlichen Fotos einem bestimmten Zeitraum zuzuordnen waren und somit das verspätete Eingreifen der Klinikärzte dokumentierten.

Martin Wortmann

\title{
Barrierefreie Praxis zahlt sich aus
}

\section{Kein Fahrstuhl, enge Gänge - Patienten mit Behinderung oder gar Rollstuhl können solche Arztpraxen nicht aufsuchen. Mit Blick schon in die nahe Zukunft sollten Praxisinhaber handeln.}

n Deutschland leben $13 \%$ der Bundesbürger mit einer amtlich anerkannten Behinderung. Das hat das Statistische Bundesamt vor einiger Zeit gemeldet. Die Tendenz ist - nicht zuletzt wegen des demografischen Wandels - steigend. Für Arztpraxen, die für alle Patienten gut erreichbar sein wollen, wird es damit immer wichtiger, darauf zu achten, die Praxis barrierefrei zu gestalten. Eine Initiative „Barrierefreie Praxis“ existiert seit dem Jahr 2009, gefördert unter anderem von der Bundesregierung.

Maßnahmen, die Barrierefreiheit fördern, sind zum Beispiel Behindertenparkplätze, ein stufenloser Eingang, beidseitig breite Handläufe, aber auch kontrastreiche Flächen mit gut lesbaren Beschriftungen und markierte Kanten zum Beispiel an Treppenstufen, die es sehbehinderten Menschen erleichtern, sich zu orientieren.

Auf Barrierefreiheit können Ärzte auch bei ihrer Website achten, damit sie für sehbehinderte Menschen zugänglich ist.

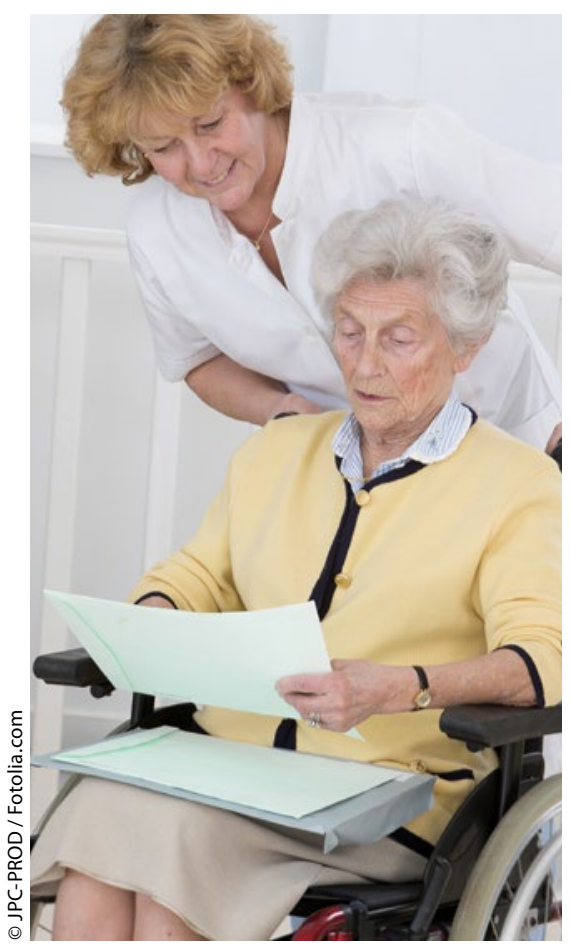

Mit dem Rollstuhl zum Arzt, das geht nur, wenn dieser eine barrierefreie Praxis hat.
Für Neubauten gelten in der Regel bereits Vorgaben für die barrierefreie Errichtung. Aber auch bei baulichen Veränderungen bestehender Gebäude kann beim zuständigen Bauamt erfragt werden, ob und welche Vorgaben der Barrierefreiheit zu beachten sind. Bei Investitionen in neue und auch in bestehende Gebäude ist die Barrierefreiheit damit immer ein Thema.

Mit Inkrafttreten des GKV-Versorgungsstärkungsgesetzes (GKV-VSG) hat dieses Thema für Arztpraxen noch zusätzlich an Bedeutung gewonnen. „Barrierefreiheit kann jetzt auch mit Blick auf die Nachbesetzung einer Praxis von Vorteil sein“, erläuterte Jessica Hanneken, Referentin für Gesundheitspolitik bei der Deutschen Apotheker- und Ärztebank (apoBank), auf Anfrage. „Der Gesetzgeber verweist darauf, dass es bei der Besetzung eines Arztsitzes ein Auswahlkriterium sein kann, ob der Nachfolger bereit ist, Maßnahmen zum Abbau von Barrieren zu ergreifen."

Investitionen in die Barrierefreiheit einer Praxis können auch unterstützend für das Marketing sein. Denn Arztsuchdienste im Internet haben diese als Kriterium aufgenommen, nach dem gesucht werden kann, zum Beispiel das weithin genutzte Portal www.arzt-auskunft.de.

Hauke Gerlof 